\title{
91. \\ SUR LE PROBLÈME DES CONTACTS.
}

[From the Journal für die reine und angewandte Mathematik (Crelle), tom. xxxIx. (1850), pp. 4-13.]

$\mathrm{J}_{\mathrm{E}}$ me propose ici la solution analytique du problème suivant:

"Étant données trois coniques inscrites à une même conique: trouver une autre conique, aussi inscrite à cette conique, qui touche les trois coniques inscrites; et tirer de là les constructions géométriques ordinaires."

Je commence par récapituler quelques-unes-des propriétés d'un système de trois coniques inscrites à la même conique.

Un système de six droites qui passent trois à trois par quatre points, s'appelle quadrangle. Les points de rencontre des côtés opposés sont les centres du quadrangle; les côtés du triangle formé par ces trois centres sont les axes du quadrangle. De même, un système de six points situés trois à trois sur quatre droites, s'appelle quadrilatère. Les droites qui passent par les angles opposés sont les axes; et les angles du triangle formé par les trois axes sont les centres du quadrilatère.

Deux coniques quelconques se coupent en quatre points qui forment un quadrangle inscrit aux deux coniques. Elles ont quatre tangentes communes qui forment un quadrilatère circonscrit aux deux coniques. Le quadrangle inscrit et le quadrilatère circonscrit ont. les même centres et les mêmes axes.

Si deux coniques sont circonscrites ou inscrites l'une à l'autre, la droite qui passe par les deux points de contact s'appelle chorde de contact, et le point de rencontre des deux tangentes communes s'appelle centre de contact.

Cela posé : les coniques circonscrites à deux coniques données, peuvent être divisées en trois classes: une conique circonscrite appartient à une quelconque de ces trois classes, selon que les points de rencontre des chordes de contact de la conique 
circonscrite et de chacune des deux coniques données coïncide avec un quelconque des trois centres du quadrangle inscrit, ou du quadrilatère circonscrit; ou, si l'on veut, selon que la droite qui passe par les centres de contact de la conique circonscrite et des deux coniques données, coïncide avec un quelconque des trois axes du quadrangle inscrit, ou du quadrilatère circonscrit.

En considérant les deux coniques données, et une conique circonscrite, nous dirons que les deux côtés du quadrangle inscrit qui se coupent dans le point d'intersection des deux chordes de contact, sont les axes de symptose des deux coniques données, et que les deux angles du quadrilatère circonscrit, situés sur la droite qui passe par les deux centres de contact, sont les centres d'homologie des deux coniques données.

Soient maintenant inscrites trois coniques à la même conique. En combinant deux à deux ces trois coniques, les six axes de symptose se couperont trois à trois en quatre points que nous nommerons centres de symptose, et les six centres d'homologie seront situés trois à trois sur quatre droites que nous appelerons axes d'homologie.

Soient

$$
U+V_{1}^{2}=0, \quad U+V_{2}^{2}=0, \quad U+V_{3}^{2}=0
$$

les équations des trois coniques inscrites, où

$$
\begin{aligned}
& U=A x^{2}+B y^{2}+C z^{2}+2 F y z+2 G x z+2 H x y, \\
& V_{1}=\alpha_{1} x+\beta_{1} y+\gamma_{2} z \\
& V_{2}=\alpha_{2} x+\beta_{2} y+\gamma_{2} z \\
& V_{3}=\alpha_{2} x+\beta_{3} y+\gamma_{3} z .
\end{aligned}
$$

$\mathrm{Si}$

$$
l x+m y+n z=0
$$

est l'équation d'une tangente commune aux coniques $U+V_{1}^{2}=0, U+V_{2}^{2}=0$, les formules de la "Note sur quelques formules \&c." [90], en adoptant la notation de cette note, donneront les équations

$$
\begin{aligned}
& \left(K+\mathfrak{A} a_{1}^{2}+\ldots\right)\left(\mathfrak{A} l^{2}+\ldots\right)-\left(\mathfrak{A} a_{1} l+\ldots\right)^{2}=0, \\
& \left(K+\mathfrak{A} a_{2}^{2}+\ldots\right)\left(\mathfrak{A} l^{2}+\ldots\right)-\left(\mathfrak{A} \alpha_{2} l+\ldots\right)^{2}=0,
\end{aligned}
$$

qui serviront à déterminer les valeurs de $l, m, n$; on obtient par là l'éxpression

$$
\sqrt{ }\left(K+\mathfrak{A} \alpha_{2}^{2}+\ldots\right)\left(\mathfrak{A} \alpha_{1} l+\ldots\right)-\sqrt{ }\left(K+\mathfrak{A} \alpha_{1}^{2}+\ldots\right)\left(\mathfrak{A} \alpha_{2} l+\ldots\right),
$$

qui fait voir que l'équation $l x+m y+n z=0$ est satisfaite en écrivant

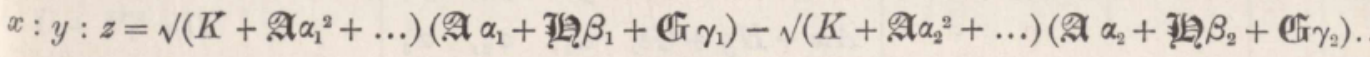

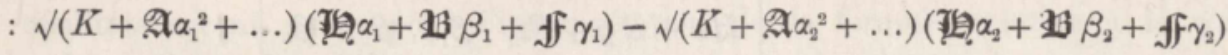

$$
\begin{aligned}
& : \sqrt{ }\left(K+\mathfrak{A} \alpha_{1}^{2}+\ldots\right)\left(\mathbb{G} \alpha_{1}+\sqrt{f} \beta_{1}+\mathbb{C} \gamma_{1}\right)-\sqrt{ }\left(K+\mathfrak{A} \alpha_{2}^{2}+\ldots\right)\left(\mathbb{G} \alpha_{2}+\sqrt{f} \beta_{2}+\mathbb{C}_{\gamma_{2}}\right) \text {, }
\end{aligned}
$$


et ces équations, qui peuvent aussi être présentées sous la forme plus simple

$$
\begin{aligned}
A x+H y+G z: & H x+B y+F z: G x+F y+C z \ldots \ldots \ldots \ldots \ldots \\
= & \sqrt{ }\left(K+\mathfrak{A} \alpha_{1}{ }^{2} \ldots\right) \alpha_{2}-\sqrt{ }\left(K+\mathfrak{A} \alpha_{2}{ }^{2} \ldots\right) \alpha_{1} \\
: & \sqrt{ }\left(K+\mathfrak{A} \alpha_{1}{ }^{2} \ldots\right) \beta_{2}-\sqrt{ }\left(K+\mathfrak{A} \alpha_{2}{ }^{2} \ldots\right) \beta_{1} \\
: & \sqrt{ }\left(K+\mathfrak{A} \alpha_{1}{ }^{2} \ldots\right) \gamma_{2}-\sqrt{ }\left(K+\mathfrak{A} \alpha_{2}{ }^{2} \ldots\right) \gamma_{1},
\end{aligned}
$$

correspondent à un centre d'homologie des deux coniques $U+V_{1}^{2}=0, U+V_{2}^{2}=0$.

En mettant, pour abréger,

$$
\sqrt{ }\left(K+\mathfrak{A} \alpha_{1}{ }^{2}+\ldots\right)=p_{1}, \quad \sqrt{ }\left(K+\mathfrak{A} \alpha_{2}{ }^{2}+\ldots\right)=p_{2}, \quad \sqrt{ }\left(K+\mathfrak{A} \alpha_{3}{ }^{2}+\ldots\right)=p_{3}
$$

on obtient facilement, pour un des axes d'homologie des trois coniques, l'équation

$$
\left|\begin{array}{llll} 
& p_{1}, & p_{2}, & p_{3} \\
A x+H y+G z, & \alpha_{1}, & \alpha_{2}, & \alpha_{3} \\
H x+B y+F z, & \beta_{1}, & \beta_{2}, & \beta_{3} \\
G x+F y+C z, & \gamma_{1}, & \gamma_{2}, & \gamma_{3}
\end{array}\right|=0
$$

et celles des trois autres axes d'homologie en peuvent être tirées en changeant les signes de $p_{1}, p_{2}, p_{3}$.

Remarquons que l'équation

$$
\left|\begin{array}{cccc}
A x+H y+G z, & 1, & 1, & 1 \\
H x+B y+F z, & \alpha_{1}, & \beta_{2}, & \beta_{3} \\
G x+F y+C z, & \gamma_{1}, & \gamma_{2}, & \gamma_{3}
\end{array}\right|=0
$$

est celle de la polaire d'un des centres de symptose des trois coniques par rapport à la conique circonscrite $U=0$, savoir de celle qui est donnée par le système $V_{1}=V_{2}=V_{3}$, et que les équations des trois autres polaires correspondantes se trouveront en changeant les signes de $\alpha_{1}, \beta_{1}, \gamma_{1}$, ou de $\alpha_{2}, \beta_{2}, \gamma_{2}$, ou de $\alpha_{3}, \beta_{3}, \gamma_{3}$.

Cherchons le pôle de l'axe d'homologie dont nous venons de trouver l'équation, par rapport à une quatrième conique inscrite $U+V^{2}=0(V=\alpha x+\beta y+\gamma z)$. En exprimant cette équation par $l x+m y+n z=0$, on obtiendra les coordonnées de ce pôle au moyen de l'équation

$$
K(\lambda X+\mu Y+\nu Z)=p^{2}(\mathfrak{A} l \lambda+\ldots)-(\mathfrak{A} \alpha \lambda+\ldots)(\mathfrak{A} \alpha l+\ldots)
$$

où, pour abréger, on a mis $p=\sqrt{ }\left(K+\mathfrak{A} \alpha^{2}+\ldots\right)$. (Mémoire cité; équation (12).) Mais ici on a

$$
l x+m y+n z=\left|\begin{array}{llll} 
& p_{1}, & p_{2}, & p_{3} \\
A x+H y+G z, & \alpha_{1}, & \alpha_{2}, & \alpha_{3} \\
H x+B y+F z, & \beta_{1}, & \beta_{2}, & \beta_{3} \\
G x+F y+C z, & \gamma_{1}, & \gamma_{2}, & \gamma_{3}
\end{array}\right|
$$


ce qui donne immédiatement

$$
\mathfrak{A} l \lambda+\ldots=K\left|\begin{array}{cccc} 
& p_{1}, & p_{2}, & p_{3} \\
\lambda, & \alpha_{1}, & \alpha_{2}, & \alpha_{3} \\
\mu, & \beta_{1}, & \beta_{2}, & \beta_{3} \\
\nu, & \gamma_{1}, & \gamma_{2}, & \gamma_{3}
\end{array}\right|, \quad \mathfrak{A} \alpha l+\ldots=K\left|\begin{array}{cccc} 
& p_{1}, & p_{2}, & p_{3} \\
\alpha, & \alpha_{1}, & \alpha_{2}, & \alpha_{3} \\
\beta, & \beta_{1}, & \beta_{2}, & \beta_{3} \\
\gamma, & \gamma_{1}, & \gamma_{2}, & \gamma_{3}
\end{array}\right|
$$

et de là on obtient

$$
\lambda X+\mu Y+\nu Z=p^{2}\left|\begin{array}{cccc} 
& p_{1}, & p_{2}, & p_{3} \\
\lambda, & \alpha_{1}, & \alpha_{2}, & \alpha_{3} \\
\mu, & \beta_{1}, & \beta_{2}, & \beta_{3} \\
\nu, & \gamma_{1}, & \gamma_{2}, & \gamma_{3}
\end{array}\right|-(\mathbb{A} \alpha \lambda+\ldots)\left|\begin{array}{cccc}
p_{1}, & p_{2}, & p_{3} \\
\alpha, & \alpha_{1}, & \alpha_{2}, & \alpha_{3} \\
\beta, & \beta_{1}, & \beta_{2}, & \beta_{3} \\
\gamma, & \gamma_{1}, & \gamma_{2}, & \gamma_{3}
\end{array}\right|
$$

savoir, en considérant cette équation comme identique par rapport à $\lambda, \mu$, $\nu$, on obtient les coordonnées $X, Y, Z$ du point dont il s'agit. En prenant particulièrement ce pôle par rapport à la conique $U+V_{2}^{2}=0$, cette équation se réduit à

$$
\frac{1}{p_{1}}(\lambda X+\mu Y+\nu Z)=p_{1}\left|\begin{array}{llll} 
& p_{1}, & p_{2}, & p_{3} \\
\lambda, & \alpha_{1}, & \alpha_{2}, & \alpha_{3} \\
\mu, & \beta_{1}, & \beta_{2}, & \beta_{3} \\
\nu, & \gamma_{1}, & \gamma_{2}, & \gamma_{3}
\end{array}\right|-\left(\boldsymbol{A} \alpha_{1} \lambda+\ldots\right) \mid \begin{array}{ccc}
\alpha_{1}, & \alpha_{2}, & \alpha_{3} \\
\beta_{1}, & \beta_{2}, & \beta_{3} \\
\gamma_{1}, & \gamma_{2}, & \gamma_{3}
\end{array}
$$

où, si l'on veut, $X, Y, Z$ seront déterminés par les expressions

$$
\begin{aligned}
& \alpha_{1} X+\beta_{1} Y+\gamma_{1} Z=-p_{1}{ }^{2}+\mathfrak{A} \alpha_{1}^{2}+\ldots,=-K \\
& \alpha_{2} X+\beta_{2} Y+\gamma_{2} Z=-p_{1} p_{2}+\mathfrak{A} \alpha_{1} \alpha_{2}+\ldots, \\
& \alpha_{3} X+\beta_{3} Y+\gamma_{3} Z=-p_{1} p_{3}+\mathbb{A} \alpha_{1} \alpha_{3}+\ldots,
\end{aligned}
$$

Le facteur $\frac{1}{p_{1}}$ a été supprimé. De là on obtient aussi l'équation de la droite menée par ce point, savoir par le pôle de l'axe d'homologie par rapport à la conique $U+V_{1}^{2}=0$, et par le centre de symptose $V_{1}=V_{2}=V_{3}$. En effet, cette équation est

$$
\left|\begin{array}{ccc}
V_{1}, & V_{2}, & V_{3} \\
1, & 1, & 1 \\
p_{1}^{2}-\mathbf{A} \alpha_{1}^{2}-\ldots, & p_{1} p_{2}-\mathbf{A} \alpha_{1} \alpha_{2}-\ldots, & p_{1} p_{3}-\mathbf{A} \alpha_{1} \alpha_{3}-\ldots
\end{array}\right|=0
$$

On pourrait également chercher le point d'intersection de la polaire du centre de symptose $V_{1}=V_{2}=V_{3}$ par rapport à $U+V_{1}^{2}=0$, et de l'axe d'homologie; ce point serait évidemment le pôle de la droite exprimée par la dernière équation, par rapport à $U+V_{1}^{2}=0$.

Ces résultats seront utiles pour l'interprétation de la formule relative à la conique qui touche les trois coniques données et que nous irons chercher maintenant. 
Représentons par $U+V^{2}=0$ l'équation de cette conique; l'équation (10) du mémoire cité donnera les expressions

$$
\left.\begin{array}{l}
\mathfrak{A} \alpha \alpha_{1}+\ldots=-K+p p_{1}, \\
\mathfrak{A} \alpha \alpha_{2}+\ldots=-K+p p_{2}, \\
\mathfrak{A} \alpha \alpha_{3}+\ldots=-K+p p_{3}
\end{array}\right\}
$$

auxquelles nous ajouterons l'équation qui donne la valeur de $p$, savoir

$$
\mathfrak{A} \alpha^{2}+\ldots=-K+p^{2}
$$

Il n'y a qu'à substituer dans cette dernière équation les valeurs de $\alpha, \beta, \gamma$ que donnent les trois autres. Par là on obtient, pour déterminer $p$, une équation du second degré et de la forme

$$
K^{2} L-2 K p M+p^{2} N=0
$$

c'est-à-dire, en faisant $M^{2}-N L=\Omega^{2}, \Lambda=\frac{L}{\Omega+M}$, on aura $p=\Lambda K$ et de là

$$
\left.\begin{array}{l}
\mathbb{A} \alpha \alpha_{1}+\ldots=-K\left(\Lambda p_{1}-1\right), \\
\mathbb{A} \alpha \alpha_{2}+\ldots=-K\left(\Lambda p_{2}-1\right) \\
\mathbb{A} \alpha \alpha_{3}+\ldots=-K\left(\Lambda p_{3}-1\right)
\end{array}\right\}
$$

où $\Lambda$ est une quantité connue, dont la valeur sera donnée dans la suite. Pour le moment il suffit de remarquer qu'en changeant_à la fois les signes de $p_{1}, p_{2}, p_{3}, \Omega$, cette quantité $\Lambda$ ne change que de signe. Au lieu de chercher les valeurs de $\alpha, \beta, \gamma$, il vaut mieux éliminer ces quantités entre ces dernières équations et l'équation $V=\alpha x+\beta y+\gamma z=0$. Cela donne, pour trouver $V$, l'équation

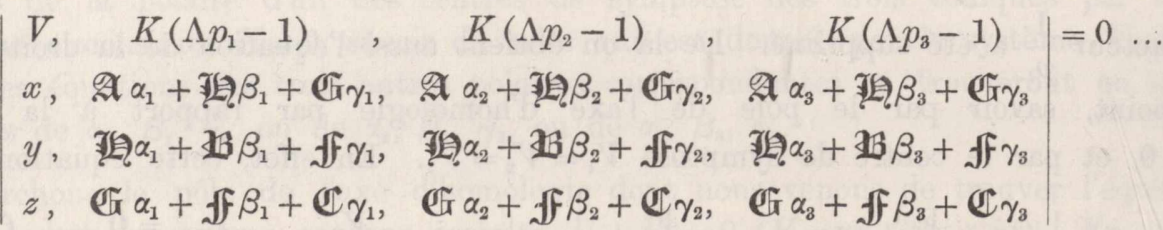

qui peut aussi être écrite comme suit:

$$
\left|\begin{array}{cccc}
V, & \Lambda p_{1}-1, & \Lambda p_{2}-1, & \Lambda p_{3}-1 \\
A x+H y+G z, & \alpha_{1}, & \alpha_{2}, & \alpha_{3} \\
H x+B y+F z, & \beta_{1}, & \beta_{2}, & \beta_{3} \\
G x+F y+C z, & \gamma_{1}, & \gamma_{2}, & \gamma_{3}
\end{array}\right|=0
$$

En mettant $V=0$, on aura l'équation de la chorde de contact de la conique cherchée et de la conique circonscrite $U=0$. En remarquant que l'équation de la 
conique cherchée peut être mise sous la forme $V=\sqrt{ }-U$, l'équation de cétte conique se présentera sous la forme très simple:

$$
\left|\begin{array}{cccc}
\sqrt{ }-U, & \Lambda p_{1}-1, & \Lambda p_{2}-1, & \Lambda p_{3}-1 \\
A x+H y+G z, & \alpha_{1}, & \alpha_{2}, & \alpha_{3} \\
H x+B y+F z, & \beta_{1}, & \beta_{2}, & \beta_{3} \\
G x+F y+C z, & \gamma_{1}, & \gamma_{2}, & \gamma_{3}
\end{array}\right|=0 \ldots \ldots \ldots \ldots . . .
$$

ou enfin, si l'on veut, sous la forme plus usitée:

$$
\left|\begin{array}{ccc}
\alpha_{1}, & \alpha_{2}, & \alpha_{3} \\
\beta_{1}, & \beta_{2}, & \beta_{3} \\
\gamma_{1}, & \gamma_{2}, & \gamma_{3}
\end{array}\right|^{2} U+\left|\begin{array}{cccc} 
& \Lambda p_{1}-1, & \Lambda p_{2}-1, & \Lambda p_{3}-1 \\
A x+H y+G z, & \alpha_{1}, & \alpha_{2}, & \alpha_{3} \\
H x+B y+F z, & \beta_{1}, & \beta_{2}, & \beta_{3} \\
G x+F y+C z, & \gamma_{1}, & \gamma_{2}, & \gamma_{3}
\end{array}\right|^{2}=0 \ldots(18)
$$

la premiere de ces deux formes est peut-être la plus élégante.

Les propriétés géométriques sont absolument indépendantes de la valeur de la quantité $\Lambda$ : mais' pour compléter la solution, je vais donner l'expression de cette quantité. Pour cela, remarquons qu'en mettant pour abréger:

$$
\begin{aligned}
& \quad \Pi=\left|\begin{array}{ccc}
\alpha_{1}, & \alpha_{2}, & \alpha_{3} \\
\beta_{1}, & \beta_{2}, & \beta_{3} \\
\gamma_{1}, & \gamma_{2}, & \gamma_{3}
\end{array}\right| \\
& l_{1}=\beta_{2} \gamma_{3}-\beta_{3} \gamma_{2}, \quad l_{2}=\beta_{3} \gamma_{1}-\beta_{1} \gamma_{3}, \ldots \ldots \ldots \ldots \ldots \ldots \ldots \ldots \\
& m_{1}=\gamma_{2} \alpha_{3}-\gamma_{3} \alpha_{2}, \\
& \vdots \\
& \lambda=l_{1}\left(p p_{1}-K\right)+l_{2}\left(p p_{2}-K\right)+l_{3}\left(p p_{3}-K\right),
\end{aligned}
$$

on aura d'abord l'équation identique

$$
-K \Pi(\alpha x+\beta y+\gamma z)=\left|\begin{array}{cccc}
p p_{1}-K, & p p_{2}-K, & p p_{3}-K \\
A x+H y+G z, & \alpha_{1}, & \alpha_{2}, & \alpha_{3} \\
H x+B y+F z, & \beta_{1}, & \beta_{2}, & \beta_{3} \\
G x+F y+C z, & \gamma_{1}, & \gamma_{2}, & \gamma_{3}
\end{array}\right|,
$$

ou, ce qui est la même chose,

$$
K \Pi(\alpha x+\beta y+\gamma z)=\lambda(A x+H y+G z)+\mu(H x+B y+F z)+\nu(G x+F y+C z) .
$$

Cela donne

$$
\Pi\left(\mathbb{A} \alpha^{2}+\ldots\right)=\lambda \alpha+\mu \beta+\nu \gamma, \quad K \Pi(\lambda \alpha+\mu \beta+\nu \gamma)=\left(A \lambda^{2}+\ldots\right),
$$


c'est-à-dire

$$
K \Pi^{2}\left(\mathfrak{A} \alpha^{2}+\ldots\right)=A \lambda^{2}+\ldots,
$$

et, en vertu de cette expression, l'équation qui sert à déterminer $p$ se réduit à

$$
K \Pi^{2}+A \lambda^{2}+\ldots-K \Pi^{2} p^{2}=0
$$

En la comparant avec l'équation $K^{2} L-2 K p M+p^{2} N=0$, on obtient

$$
\left.\begin{array}{lc}
L= & \Pi^{2}+\left[A\left(l_{1}+l_{2}+l_{3}\right)^{2}+\ldots\right], \\
M= & {\left[A\left(l_{1}+l_{2}+l_{3}\right)\left(l_{1} p_{1}+l_{2} p_{2}+l_{3} p_{3}\right)+\ldots\right],} \\
N= & K \Pi^{2}+\left[A\left(l_{1} p_{1}+l_{2} p_{2}+l_{3} p_{3}\right)^{2}+\ldots\right],
\end{array}\right\}
$$

et de là, par une transformation déjà employée,

$$
M^{2}-N L=\Omega^{2}=\Pi^{2}\left\{\begin{array}{l}
-\left[A\left(l_{1} p_{1}+l_{2} p_{2}+l_{3} p_{3}\right)^{2}+\ldots\right] \\
+K\left[A\left(l_{1}+l_{2}+l_{3}\right)^{2}+\ldots\right]+K \Pi^{2} \\
-\left[\boldsymbol{A}\left\{p_{1}\left(\alpha_{2}-\alpha_{3}\right)+p_{2}\left(\alpha_{3}-\alpha_{1}\right)+p_{3}\left(\alpha_{1}-\alpha_{2}\right)\right\}^{2}+\ldots\right]
\end{array}\right\} \ldots(22)
$$

mais l'interpretation de ce résultat paraît être difficile.

En revenant sur l'équation trouvée pour la conique qui touche les trois coniques données, remarquons que les signes de $\alpha_{1}, \beta_{1}, \gamma_{1}$, ou de $\alpha_{2}, \beta_{2}, \gamma_{2}$, ou de $\alpha_{3}, \beta_{3}, \gamma_{3}$, peuvent être changés conjointement. Cela revient en effet à écrire $-V_{1}$, ou $-V_{2}$, ou $-V_{3}$ au lieu de $+V_{1}$, ou de $+V_{2}$, ou de $+V_{3}$, ce qui ne change pas les coniques inscrites. Mais il est facile de voir qu'en changeant à la fois les signes de $V_{1}, V_{2}, V_{3}$, on ne change pas l'équation de la conique dont il s'agit; cette équation ne change non plus, en changeant à la fois les signes de $p_{1}, \bar{p}_{2}, p_{3}, \Omega$; de manière que l'équation trouvée correspond réellement à 32 coniques différentes. En distinguant ces 32 coniques par des symboles de la forme

$$
\left( \pm V_{1}, \pm V_{2}, \pm V_{3}, \pm p_{1}, \pm p_{2}, \pm p_{3}, \pm \Omega\right):
$$

quatre symboles tels que

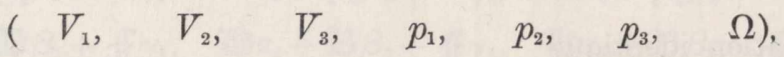

$$
\begin{aligned}
& \left(-V_{1}, \quad-V_{2}, \quad-V_{3}, \quad p_{1}, \quad p_{2}, \quad p_{3}, \quad \Omega\right),
\end{aligned}
$$

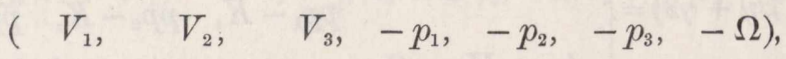

$$
\begin{aligned}
& \left(-V_{1}, \quad-V_{2}, \quad-V_{3}, \quad-p_{1}, \quad-p_{2}, \quad-p_{3}, \quad-\Omega\right),
\end{aligned}
$$

ne se rapporteront qu'à une seule conique. Nous appelerons paires de coniques deux coniques quelconques exprimées par des symboles de la forme

$$
\left(V_{1}, V_{2}, V_{3}, p_{1}, p_{2}, p_{3}, \pm \Omega\right) ;
$$

donc les 32 coniques forment 16 paires, groupées quatre à quatre de deux manières différentes: savoir, pour former un groupe, quatre paires telles que

$$
\left( \pm V_{1}, \pm V_{2}, V_{3}, p_{1}, p_{2}, p_{3}, \pm \Omega\right)
$$


ou quatre paires telles que

$$
\left(V_{1}, V_{2}, V_{3}, \pm p_{1}, \pm p_{2}, p_{3}, \pm \Omega\right)
$$

peuvent être combinées. Ces deux espèces de groupes peuvent être distinguées par les noms groupes par rapport d̀ un axe d'homologie, et groupes par rapport à un centre de symptose. En effet: considérons une paire de coniques, par exemple celle qui est représentée par les symboles $\left(V_{1}, V_{2}, V_{3}, p_{1}, p_{2}, p_{3}, \pm \Omega\right)$. Les équations des deux coniques de la paire sont les mêmes aux valeurs de $\Lambda$ près; il est donc évident que les chordes de contact de ces deux coniques avec la conique circonscrite $U=0$ doivent se rencontrer au point d'intersection des droites

$$
\left|\begin{array}{llll}
A x+H y+G z, & 1, & 1, & 1 \\
H x+B y+F z, & \beta_{1}, & \beta_{2}, & \beta_{3} \\
G x+F y+C z, & \gamma_{1}, & \gamma_{2}, & \gamma_{3}
\end{array}\right|=0,\left|\begin{array}{llll} 
& p_{1}, & p_{2}, & p_{3} \\
A x+H y+G z, & \alpha_{1}, & \alpha_{2}, & \alpha_{3} \\
H x+B y+F z, & \beta_{1}, & \beta_{2}, & \beta_{3} \\
G x+F y+C z, & \gamma_{1}, & \gamma_{2}, & \gamma_{3}
\end{array}\right|=0
$$

La première de ces équations se rapporte à la polaire d'un des centres de symptose des trois coniques inscrites par rapport à la conique circonscrite; la seconde se rapporte à un des axes d'homologie. On a donc le théorème suivant:

"Les points de rencontre des 16 chordes de contact des paires de coniques sont les points de rencontre des polaires des quatre centres de symptose par rapport à la conique circonscrite, avec les quatre axes d'homologie."

Cela suffit pour expliquer la manière dont les deux espèces de groupes ont été distinguées.

Cherchons l'équation de la droite menée par les points de contact d'une des coniques inscrites (par exemple celle que donne l'equation $U+V_{1}^{2}=0$ ) avec deux coniques de la même paire. En représentant par

$$
U+(\alpha x+\beta y+\gamma z)^{2}=0 \text { et } U+\left(\alpha^{\prime} x+\beta^{\prime} y+\gamma^{\prime} z\right)^{2}=0
$$

les équations de ces deux coniques: les équations des tangentes communes seront

$$
\left(\alpha-\alpha_{1}\right) x+\left(\beta-\beta_{1}\right) y+\left(\gamma-\gamma_{1}\right) z=0 \text { et }\left(\alpha^{\prime}-\alpha_{1}\right) x+\left(\beta^{\prime}-\beta_{1}\right) y+\left(\gamma^{\prime}-\gamma_{1}\right) z=0,
$$

et l'équation de la droite qui passe par les points de contact de ces deux droites avec la conique $U+V_{1}^{2}=0$ est:

$$
\begin{aligned}
& A\left(\beta \gamma^{\prime}-\beta^{\prime} \gamma\right) x+\ldots+A\left(\gamma_{1}\left(\beta^{\prime}-\beta\right)-\beta_{1}\left(\gamma^{\prime}-\gamma\right)\right) x+\ldots \\
& \quad+\left(\alpha_{1} x+\beta_{1} y+\gamma_{1} z\right)\left[\alpha_{1}\left(\beta \gamma^{\prime}-\beta^{\prime} \gamma\right)+\beta_{1}\left(\gamma \alpha^{\prime}-\gamma^{\prime} \alpha\right)+\gamma_{1}\left(\alpha \beta^{\prime}-\alpha^{\prime} \beta\right)\right]=0
\end{aligned}
$$

Pour réduire cette équation, en exprimant par $\Pi, l_{1}, m_{1}$ \&c. les valeurs plus haut, mettons $\lambda=l_{1}\left(\Lambda p_{1}-1\right)+l_{2}\left(\Lambda p_{2}-1\right)+l_{3}\left(\Lambda p_{3}-1\right)$ \&c., et soient $\lambda^{\prime}, \mu^{\prime}, \nu^{\prime}$ ce que deviennent les valeurs de $\lambda, \mu, \nu$ en écrivant $\Lambda^{\prime}$ au lieu de $\Lambda$. On aura

$$
\Pi \alpha=A \lambda+H \mu+G \nu, \& c .
$$

C. 
et des expressions pareilles des valeurs de $\lambda^{\prime}, \mu^{\prime}, \nu^{\prime}$. Delà on tire

$$
\begin{aligned}
& \Pi^{2}\left(\beta \gamma^{\prime}-\beta^{\prime} \gamma\right)=\left(\mu \nu^{\prime}-\mu^{\prime} \nu\right) \mathfrak{A}+\left(\nu \lambda^{\prime}-\nu^{\prime} \lambda\right) \mathfrak{I}+\left(\lambda \mu^{\prime}-\lambda^{\prime} \mu\right) \mathbb{G}, \\
& \text { \&c., } \\
& \mu \nu^{\prime}-\mu^{\prime} \nu=\left(m_{2} n_{3}-m_{3} n_{2}\right)\left\{\left(\Lambda p_{2}-1\right)\left(\Lambda^{\prime} p_{3}-1\right)-\left(\Lambda^{\prime} p_{2}-1\right)\left(\Lambda p_{3}-1\right)\right\}+\& c . \\
& =\Pi\left(\Lambda^{\prime}-\Lambda\right)\left[\alpha_{1}\left(p_{2}-p_{3}\right)+\alpha_{2}\left(p_{3}-p_{1}\right)+\alpha_{3}\left(p_{1}-p_{2}\right)\right],
\end{aligned}
$$

c'est-à-dire, en supprimant le facteur commun $\frac{\Lambda^{\prime}-\Lambda}{\Pi}$ :

$$
\begin{aligned}
& \left.\beta \gamma^{\prime}-\beta^{\prime} \gamma=\left(\mathfrak{A} \alpha_{1}+\right]_{-1} \beta_{1}+\boldsymbol{G} \gamma_{1}\right)\left(p_{2}-p_{3}\right) \\
& +\left(\mathbb{A} \alpha_{2}+\text { 政 } \beta_{2}+\mathbb{G} \gamma_{2}\right)\left(p_{3}-p_{1}\right) \\
& +\left(\boldsymbol{A} \alpha_{3}+\mathfrak{I}_{-} \beta_{3}+\mathfrak{G} \gamma_{3}\right)\left(p_{1}-p_{2}\right) \text {, }
\end{aligned}
$$

\&c.

Aussi on aura, en supprimant le même facteur:

$$
\begin{aligned}
\gamma_{1}\left(\beta^{\prime}-\beta\right)-\beta_{1}\left(\gamma^{\prime}-\gamma\right)= & \left(l_{1} p_{1}+l_{2} p_{2}+l_{3} p_{3}\right)\left(\gamma_{1} H-\beta_{1} G\right) \\
& +\left(m_{1} p_{1}+m_{2} p_{2}+m_{3} p_{3}\right)\left(\alpha_{1} G-\gamma_{1} A\right) \\
& +\left(n_{1} p_{1}+n_{2} p_{2}+n_{3} p_{3}\right)\left(\beta_{1} A-\alpha_{1} H\right) .
\end{aligned}
$$

De là on obtient immédiatement

$$
A\left(\beta \gamma^{\prime}-\beta^{\prime} \gamma\right) x+\ldots=K\left[V_{1}\left(p_{2}-p_{3}\right)+V_{2}\left(p_{3}-p_{1}\right)+V_{3}\left(p_{1}-p_{2}\right)\right],
$$

et, par une réduction un peu plus difficile,

$A\left(\gamma_{1}\left(\beta^{\prime}-\beta\right)-\beta_{1}\left(\gamma^{\prime}-\gamma\right)\right) x+\ldots$

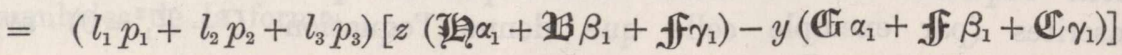

$$
\begin{aligned}
& \left.+\left(m_{1} p_{1}+m_{2} p_{2}+m_{3} p_{3}\right)\left[x\left(\mathfrak{G} \alpha_{1}+\sqrt{f} \beta_{1}+\mathfrak{C}_{\gamma_{1}}\right)-z\left(\mathfrak{A} \alpha_{1}+\right]_{1} \beta_{1}+\mathbb{G} \gamma_{1}\right)\right]
\end{aligned}
$$

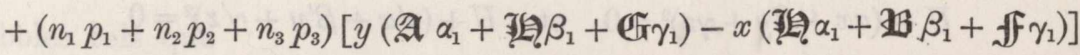

$$
\begin{aligned}
& =V_{1}\left[p_{2}\left(\mathfrak{A} \alpha_{1} \alpha_{3}+\ldots\right)-p_{3}\left(\mathfrak{A} \alpha_{1} \alpha_{2}+\ldots\right)\right]+V_{2}\left[p_{3}\left(\mathfrak{A} \alpha_{1}^{2}+\ldots\right)-p_{1}\left(\mathfrak{A} \alpha_{1} \alpha_{3}+\ldots\right)\right] \\
& +V_{3}\left[p_{1}\left(\mathfrak{A} \alpha_{1} \alpha_{2}+\ldots\right)-p_{2}\left(\mathfrak{A} \alpha_{1}^{2}+\ldots\right)\right]
\end{aligned}
$$

et enfin

$$
\begin{aligned}
& \left(\alpha_{1} x+\beta_{1} y+\gamma_{1} z\right)\left[\alpha_{1}\left(\beta \gamma^{\prime}-\beta^{\prime} \gamma\right)+\beta_{1}\left(\gamma^{\prime}-\gamma^{\prime} \alpha\right)+\gamma_{1}\left(\alpha \beta^{\prime}-\alpha^{\prime} \beta\right)\right] \\
& =V_{1}\left[\mathfrak{A}\left(\alpha_{1}{ }^{2}+\ldots\right)\left(p_{2}-p_{3}\right)+\left(\mathfrak{A} \alpha_{1} \alpha_{2}+\ldots\right)\left(p_{3}-p_{1}\right)+\left(\mathfrak{A} \alpha_{1} \alpha_{3}+\ldots\right)\left(p_{1}-p_{2}\right)\right]
\end{aligned}
$$

Donc, en réunissant ces expressions des trois parties de l'équation dont il s'agit, cette équation se réduit à

$$
\begin{aligned}
& V_{1}\left[\left(K+\mathfrak{A} \alpha_{1}{ }^{2}+\ldots\right)\left(p_{2}-p_{3}\right)-\left(\mathfrak{A} \alpha_{1} \alpha_{2}+\ldots\right) p_{1}+\left(\mathfrak{A} \alpha_{1} \alpha_{3}+\ldots\right) p_{1}\right] \\
& +V_{2}\left[\left(K+\mathfrak{A} \alpha_{1}^{2}+\ldots\right) p_{3}-\left(K+\mathfrak{A} \alpha_{1}^{2}+\ldots\right) p_{1}\right] \\
& +V_{3}\left[\left(K+\mathfrak{A} \alpha_{1} \alpha_{2}+\ldots\right) p_{1}-\left(K+\mathfrak{A} \alpha_{1}^{2}+\ldots\right) p_{2}\right]=0
\end{aligned}
$$


En mettant $p_{1}{ }^{2}$ au lieu de $K+\mathcal{A} \alpha_{1}{ }^{2}+\ldots$ et en supprimant le facteur commun $p_{1}$, on aura

$$
\begin{aligned}
V_{1}\left[p_{1} p_{2}-\left(\mathfrak{A} \alpha_{1} \alpha_{2}+\ldots\right)-p_{1} p_{3}+\left(\mathfrak{A} \alpha_{1} \alpha_{3}+\ldots\right)\right]+V_{2}[ & \left.p_{1} p_{3}-\left(\mathfrak{A} \alpha_{1} \alpha_{3}+\ldots\right)-K\right] \\
& +V_{3}\left[K-p_{1} p_{2}+\left(\mathfrak{A} \alpha_{1} \alpha_{2}+\ldots\right)\right]=0,
\end{aligned}
$$

et en remettant $p_{1}{ }^{2}-\left(\mathfrak{A} \alpha_{1}^{2}+\ldots\right)=K$, on obtient pour l'équation dont il s'agit :

$$
\left|\begin{array}{ccc}
V_{1}, & V_{2}, & V_{3} \\
1, & 1, & 1 \\
p_{1}^{2}-\left(\mathfrak{A} \alpha_{1}^{2}+\ldots\right), & p_{1} p_{2}-\left(\mathfrak{A} \alpha_{1} \alpha_{2}+\ldots\right), & p_{1} p_{3}-\left(\mathfrak{A} \alpha_{1} \alpha_{3}+\ldots\right)
\end{array}\right|=0 \ldots
$$

Cette équation est celle de la droite menée par les points de contact de deux coniques de la même paire avec la conique $U+V_{1}^{2}=0$. Elle est la même qui a été déjà obtenue pour la droite résultante d'une certaine construction géométrique; on est donc arrivé au théorème connu suivant:

"La droite menée par le pôle d'un axe d'homologie par rapport à une des trois coniques inscrites, et par un centre de symptose, rencontre cette même conique en deux points qui sont les points de contact de cette conique avec deux coniques de la même paire ;"

ou, ce qui revient au même:

"Le point de rencontre de la polaire d'un centre de symptose, par rapport à une des trois coniques inscrites, et d'un axe d'homologie, est le point de rencontre des tangentes communes de cette même conique et de deux coniques de la même paire." 